\title{
Civilisations
}

Revue internationale d'anthropologie et de sciences

humaines

$61-2 \mid 2013$

Chamanismes en mouvement

\section{Chamanisme et itinérance}

Réflexions sur les réseaux chamaniques qui relient l'Europe et l'Amérique du Sud

\section{Silvia Mesturini Cappo}

\section{OpenEdition}

\section{Journals}

Édition électronique

URL : http://journals.openedition.org/civilisations/3256

DOI : 10.4000/civilisations.3256

ISSN : 2032-0442

\section{Éditeur}

Institut de sociologie de l'Université Libre de Bruxelles

\section{Édition imprimée}

Date de publication : 28 juin 2013

Pagination : 107-122

ISSN : 0009-8140

Référence électronique

Silvia Mesturini Cappo, «Chamanisme et itinérance », Civilisations [En ligne], 61-2 | 2013, mis en ligne le 28 juin 2016, consulté le 01 mai 2019. URL : http://journals.openedition.org/civilisations/3256 ;

DOI : 10.4000/civilisations.3256 


\title{
Chamanisme et itinérance
}

\author{
Réflexions sur les réseaux chamaniques qui relient l'Europe \\ et l'Amérique du Sud
}

\author{
Silvia MESTURINI CAPPO
}

Résumé : Cet article propose une réflexion autour de divers terrains ethnographiques réalisés entre 2004 et 2010 dans des lieux considérés comme emblématiques par un chamanisme récemment internationalisé et très mobile. En explorant les défis théoriques et idéologiques qui bipolarisent le champ discursif du chamanisme contemporain, le texte présente les tendances universalistes propres à un chamanisme internationalisé qui relie l'Amérique du Sud et l'Europe et les points de vue académiques qui s'inscrivent en faux contre une telle perspective. Le chamanisme apparaîtra ainsi comme un phénomène mouvant et multiforme qui ne peut ni être rangé dans un discours de fidélité absolue à des traditions ancestrales, ni clairement scindé en un chamanisme " traditionnel » et un néo-chamanisme "inventé de toutes pièces ». La relation existant entre chamanisme et itinérance est au centre du propos. En présentant les étapes et les résultats d'une ethnographie itinérante, l'article argumente l'hypothèse d'une continuité de l'itinérance qui relierait les chamanismes d'hier et d'aujourd 'hui, les formes plus locales, vernaculaires, et celles soutenues par la circulation internationale des pratiques rituelles et des praticiens.

Mots-clés : ethnographie multi-située, réseaux chamaniques, itinérance, continuité.

Summary: The argument of this article is based on multi-sited fieldwork conducted between 2004 and 2010 in different localities considered "emblematic" by internationalized and mobile forms of contemporary shamanism. By exploring the theoretical and ideological issues that bipolarize contemporary shamanic discourse, this text shall present both the universalist tendencies that, through the internationalisation of shamanism, connect the South American continent and Europe, and the academic responses that differ from these. Shamanism will appear as a multiple and mobile phenomenon that cannot be assimilated to neither an absolute faithfulness towards ancestral traditions, nor to a thoroughly invented neo-shamanism. What ties shamanism and itinerancy will be at the core of our argument. After presenting the stages and results of an itinerant ethnography, this article will contend that itinerancy allows for an hypothesis of continuity that links contemporary and past shamanisms, and connects more vernacular and local forms to those supported by the international circulation of practitioners and ritual practice.

Keywords: Multi-sited ethnography, shamanic networks, itinerancy, continuity. 


\section{Du chamanisme et de ses usages}

État épileptoïde, hystérique, ou au contraire parfaite normalité adaptée aux règles d'une culture; sorcellerie, ou au contraire psychothérapie avant la lettre; magie agressive, ou au contraire système religieux; simple technique extatique, ou au contraire système de pensée; institution centrale et unificatrice d'ethnies morcelées par le nomadisme et la dispersion territoriale, ou au contraire pratique semi-clandestine et marginale à l'intérieur de sociétés étatisées, parent pauvre d'une religion dominante; variante de la possession, ou au contraire démarche symétriquement inverse de contact avec le surnaturel ? Le chamanisme et les chamanes semblent avoir mis à rude épreuve les concepts et les classifications des sciences humaines. (Losonczy 1990:75)

En mettant à l'épreuve nos concepts et nos classifications, les études du chamanisme semblent nous renvoyer systématiquement aux défis multiples de la description et de la compréhension de l'Autre, au point de devenir, aux yeux de Roberte Hamayon, une sorte d' « outil de pensée » (2003:7-54). Nombreuses ont été les façons d'aborder la figure emblématique du chamane. Pour ne prendre que deux exemples, malade névrosé ou même psychotique dans la réflexion de Georges Devereux, il devient un psychothérapeute qui combat le désordre dans le corps en activant un système mythique complexe dans celle de Claude Lévi-Strauss. Parallèlement, le terme s'utilise de plus en plus en sciences humaines afin d'englober " des systèmes religieux de sociétés traditionnelles restés sans nom jusqu'alors » (Hamayon 2000:9). En se substituant progressivement aux notions classiques de totémisme ou d'animisme, le chamanisme devient une catégorie polysémique, vouée à rassembler en son sein tous les exclus ou les opposants des religions instituées et des sociétés industrielles. C'est en ce sens que l'existence et l'utilisation du terme seraient le témoignage d'un rapport de force particulier, inhérent à diverses modalités de contact et forgé successivement par le point de vue du colon, du missionnaire, de l'acculturateur, de l'entrepreneur, voire de tout observateur extérieur. L'empreinte de ce regard extérieur et multiple porté sur des phénomènes rituels ponctuels a contribué à créer la catégorie " chamanisme » comme emblème d'une d'altérité exotique, « un quelque chose qui cristallise les rapports de l'Occident avec son Autre » (Hamayon $2003: 10$ ).

Le chamanisme est devenu un enjeu de débat à l'intérieur de l'anthropologie car depuis les écrits de Boas, de van Gennep puis de Lévi-Strauss, l'anthropologie s'efforce de décrire les « croyances » ou les « représentations » de l'Autre. Le chamane et le chamanisme deviennent ainsi des figures comparables à nos systèmes religieux ou à nos dispositifs de thérapie psychique ou encore à nos institutions de contrôle social.

Bien que ces différentes approches puissent en partie être réduites aux attentes et paradigmes de l'observateur, ces auteurs fournissent des descriptions du phénomène chamanique qui ne cessent de prouver l'extrême diversité des représentations et des pratiques qui caractérisent les «peuples à chamanes ", leurs pratiques et leurs discours. Nombre d'ethnologues qui avaient abordé le chamanisme comme un système culturel propre à des contextes ethniques décrits au départ comme isolés se sont ensuite penchés sur les phénomènes d'échange, d'emprunt, d'adaptation et de syncrétisme dont ces mêmes ensembles font preuve au contact avec leurs différents voisins, interlocuteurs ou envahisseurs (Chaumeil 1992; Anne-Marie Losonczy 1990, 2006 ; Thomas et Humphrey 1994). Plus récemment, des anthropologues comme Manuela Carneiro 
da Cunha (1998); Esther Jean Langdon (2007); Michael Taussig (1986) et Peter Gow $(1990,1993,1994)$ ont montré le caractère fondamentalement interethnique des chamanismes sud-américains, non seulement dans le cadre des déplacements et du métissage forcés par l'organisation coloniale mais bien avant l'arrivée des colons européens. Peter Gow (1994) et Michael Taussig (1986), en particulier, ont argumenté l'émergence d'un « chamanisme métis », postcolonial et urbain, relativement uniforme pour l'ensemble du bassin amazonien.

Si d'une part certains ethnologues mettent l'accent sur le caractère fondamentalement ouvert, interactif et malléable des systèmes chamaniques locaux, les tenants de l'approche perspectiviste, d'autre part, dont les représentants les plus célèbres sont Eduardo Viveiros de Castro, Tânia Stolze Lima et Aparecida Vilaça, cherchent, à travers une anthropologie du corps et de la personne, le dénominateur commun aux différents systèmes amérindiens locaux afin de dégager une façon de faire et de voir qui seraient à proprement parler « indigène ».

Mais, parallèlement au cheminement de la pensée anthropologique, le «chamanisme» connaît progressivement un succès qui le place au centre d'une polyphonie de discours et de pratiques qui traversent des milieux socioprofessionnels très divers.

En Europe et en Amérique du Nord d'abord, puis en Amérique latine, et contrairement aux souhaits d'une bonne partie de la communauté scientifique, le terme chamanisme quitte progressivement les réseaux académiques et commence à désigner une technique de soins particulièrement « exotique » à l'intérieur du vaste ensemble des thérapies « alternatives » ou « parallèles », désormais largement représentées et utilisées parmi les options du marché médical et paramédical contemporain. Comme c'est le cas pour une bonne partie des thérapies de cet ensemble, la frontière qui départage habituellement la médecine et le religieux est remise expressément en cause en faveur d'une approche holistique et spirituelle d'un homme en quête de soins et de développement personnel.

Objet de la démarche comparative en anthropologie, le chamanisme construit son histoire contemporaine en s'alliant progressivement à des «causes » de plus en plus variées: des thérapies spirituelles à l'écologie, des revendications indigénistes aux mouvements prophétiques millénaristes et apocalyptiques. Invoqué dans des lieux et contextes de plus en plus divers, il s'internationalise en répondant à des attentes de plus en plus globalisées et formulées dans un langage que l'on pourrait désigner comme étant de facture «New Age ${ }^{1}$. Il devient un champ d'expérimentation mystique permettant l'accès à un « autre monde »: un monde « spirituel », un monde peuplé d' " esprits », un monde perçu comme « récusé » par la culture " moderne ». Le chamanisme inspire des voyages dans des pays lointains, reconnus comme les lieux du chamanisme le plus authentique. L'Amérique latine est sans aucun doute l'un des continents privilégiés: l'Amazonie ou les déserts du nord du Mexique comptent parmi les destinations les plus prestigieuses car des pratiques reconnues comme " chamaniques » y sont associées à l'usage de substances psychoactives. Entre voyage exotique et pèlerinage spirituel, de nombreux Occidentaux s'aventurent vers ces lieux éloignés pour rencontrer sur place des infrastructures prêtes à les accueillir et à répondre à leurs attentes. Les

1. Le terme New Age renvoie à un ensemble très hétérogène de références théoriques et de pratiques, rituelles et/ou thérapeutiques, qui serait concomitant, voire synonyme, de la contre-culture nordaméricaine et européenne née dans les années 1960-1970. Ce sont surtout des historiens des religions qui se sont penchés sur la définition et la délimitation du phénomène (Hanegraaf 1996, Hammer 2001). 
routes autrefois défrichées par les hippies itinérants des années de la contre-culture semblent aujourd'hui transformées en itinéraires stabilisés pour ceux qui sont en quête d'initiation, de soins ou d'aventure spirituelle.

Parallèlement, de plus en plus d'Occidentaux revendiquent aujourd'hui le titre de chamane, le plus souvent en l'associant à d'autres qualifications indiquant une compétence thérapeutique ou spirituelle (psychothérapeute, psychanalyste, médecin, maître Reiki, Yogi, voyant, etc.), selon une logique qui valorise l'accumulation de compétences et de références liées à des systèmes médico-religieux venant d'ailleurs. Un apprentissage réalisé auprès de chamanes " ethniques » est un facteur de grand prestige, ce qui motive chez beaucoup le choix d'entreprendre des séjours prolongés dans les lieux les plus réputés du soi-disant « chamanisme authentique ». La forte valorisation des chamanes " indigènes " entraîne aussi un déplacement régulier de ceux-ci vers les capitales des pays du Nord, lieux où se rassemble la grande majorité de la clientèle du « chamanisme internationalisé ».

Conjointement à la recherche de contact avec les chamanismes « ethniques », la « renaissance »d'un chamanisme « européen » - souvent étiqueté de " druidique » ou de « celte » et voué à la défense des « médecines des campagnes de chez nous »élargit la gamme des spiritualités et des initiations à caractère thérapeutique accessibles sur le marché contemporain. Eloignées dans l'espace et dans le temps, les sources légitimes du chamanisme ont en commun d'appartenir à « l'ailleurs », au lointain, aux oubliés de l'histoire ou aux exclus de la modernité. Paradoxalement, ce chamanisme renaissant qui se veut traditionnel ne cesse de chercher reconnaissance et légitimation auprès de la science et de ses représentants.

Par conséquent, l'essor de la référence chamanique a confronté certains peuples et certains individus avec le défi d'incarner le chamanisme traditionnel dans un contexte plus large où les cultures, les ethnies et les traditions deviennent des signifiants de plus en plus mobilisés et flottants. Mais le chamanisme et son essor international participent aussi à une interconnexion de grande échelle et dessinent ainsi des itinéraires et des réseaux particuliers à l'intérieur d'une interconnectivité globale et globalisante.

\section{Une ethnographie itinérante ${ }^{2}$}

Dans le cadre du terrain multi-situé réalisé entre 2004 et 2010, entre l'Europe et l'Amérique latine, le chamanisme n'a pas été abordé comme un phénomène ayant une cohérence intrinsèque mais comme une "référence », un label, mobilisé et formaté par des interactions particulières dans des contextes multiples et variés. J'ai commencé mon terrain auprès de trois « cercles chamaniques » européens: un en Ile-de-France, un deuxième dans le nord de la Hollande et un troisième dans les Iles Canaries. J'entends par « cercles chamaniques » des petits groupes d'initiés qui se réunissent régulièrement autour d'un ou de plusieurs praticiens chamanes. Lorsqu'un chamane en particulier coordonne le groupe, d'autres chamanes peuvent être invités afin de multiplier les approches et les techniques rituelles. Si le coordinateur du groupe n'est pas un chamane, alors l'invitation de chamanes divers sera beaucoup plus systématique et plurielle.

2. La recherche ethnographique à laquelle je fais référence ici à été réalisée dans la cadre de mes recherches doctorales et a abouti à la rédaction de la thèse, en 2010 : Espaces chamaniques en mouvement. Itinéraires vécus et géographies multiples entre Europe et Amérique latine. 
Je parle de ces cercles en termes de " petits groupes » bien qu'il ne s'agisse pas de groupes fermés. Un nombre très élevé de participants irréguliers voire de participants ponctuels, réunis dans des mailing lists qui comptent plusieurs centaines de personnes, gravite autour d'un petit noyau dur, plus stable et régulier dans sa présence et dans son engagement. À partir de ces trois premières situations de terrain, l'idée d'une recherche itinérante a pris forme.

L'itinérance apparaissait comme la manière la plus reconnue et la plus privilégiée d'acquérir une compétence chamanique solide. Elle était aussi l'objet d'une analogie courante qui rapprochait le fait de se déplacer dans l'espace parmi des experts rituels divers et le fait de trouver et d'accomplir de « son propre chemin » de « réveil » et de « développement ». Le continent sud-américain était de loin la référence territoriale la plus citée parmi les lieux du chamanisme le plus traditionnel et authentique. Des contacts plus ponctuels de chamanes et de centres chamaniques particuliers m'ont été aussi livrés: certaines adresses m'étaient décrites comme des lieux de grand pouvoir et de sagesse qu'il fallait absolument que je visite afin de réaliser une bonne étude. D'autres m'étaient fortement déconseillées selon des accusations diverses allant du charlatanisme à la sorcellerie. C'est ainsi que les enjeux de réputation, les rumeurs, les recommandations et les discrédits ont dirigé ma démarche itinérante, une démarche qui se constituait au fur et à mesure sur base de recommandations et d'invitations ou, au contraire, d'interdits formels de fréquenter tel ou tel autre chamane une fois une alliance établie avec un certain expert et son réseau. Cette dynamique n'a fait que s'intensifier lorsque je suis arrivée sur le continent sud-américain où l'offre de pratiques chamaniques était beaucoup plus importante et variée.

Il est difficile de comptabiliser le nombre de lieux et d'experts rituels rencontrés sur ce terrain qui a impliqué une immersion importante dans ces réseaux qui relient de nombreux pays européens et sud-américains. Il y eut beaucoup de rencontres ponctuelles et de terrains sans retours. Ceci dit, un terrain plus approfondi fait de nombreux allersretours a été mené en explorant un réseau particulier qui, basé à Lima et constitué d'une demi-douzaine de chamanes réunis autour d'une ONG de « médecine traditionnelle », reliait la capitale du Pérou à la ville d'Iquitos en Amazonie, à celle de Cusco dans les Andes péruviennes et à des cercles de praticiens dans le sud de la France et dans le nord de l'Italie. Un séjour de plus longue durée à été réalisé dans la ville même d'Iquitos, où j'ai pu observer deux « centres chamaniques » voués à l'accueil de voyageurs étrangers, où des chamanes amazoniens travaillaient aux côtés d'initiés étrangers. Les terrains européens de longue durée, en Belgique, en France et en Espagne, se sont concentrés autour de petits groupes de praticiens qui invitaient régulièrement des chamanes sudaméricains (principalement péruviens, équatoriens et colombiens) et qui se rendaient sur le continent américain afin de poursuivre des initiations plus poussées.

Le fil conducteur de ces terrains était une interrogation sur ces modalités particulières d'action associées à l'étiquette chamanique qui se distinguaient par leur performance, leur efficacité et leur transposabilité. Le point de départ de mon étude n'était pas une définition préalable du « chamanisme » et la recherche n'a abouti à aucune définition formulée a posteriori. Par contre, les modalités interactionnelles par lesquelles des définitions se créent, se discutent et se mettent à circuler ont été au centre de mon travail ethnographique. Ce même angle d'approche a été utilisé pour les rituels, les objets rituels, les chants, les ouvrages à lire, les personnes à rencontrer, les réseaux à suivre, les sites internet à consulter, les lieux de culte à visiter. La façon dont ce paysage prenait 
forme, tenait ensemble et réagissait aux critiques, aux querelles, aux défis de ceux qui l'empruntaient et le mettaient en représentation s'est retrouvé au centre de l'analyse.

En prenant cette position en tant qu'ethnologue, j'ai pu observer comment certains praticiens cumulaient les éléments nécessaires à obtenir une réputation de chamane puissant et fiable aux yeux d'un public essentiellement urbain et issu des pays du Nord, alors que d'autres restaient confinés à une demande fortement localisée (Losonczy et Mesturini Cappo 2013, sous presse). La confrontation avec le terrain et avec ses modes de fonctionnement rendait évident que la question même de la définition de ce qui est chamanique et de ce qui ne l'est pas, de ce qui vaut le voyage et de ce qu'il faut éviter, donnait sens et structurait les discours et les pratiques des chamanes et des « chamanisants » qui partagent cet espace international. Pour le chamane voulant travailler dans les réseaux internationaux, il était indispensable de contextualiser sa propre pratique dans un discours globalisé qui faisait l'éloge de la sagesse des peuples premiers, une sagesse perdue dans un monde « occidental » fait d'industrialisation, d'individualisme et d'une exploitation sans limite des ressources naturelles. Ce discours justifiait et motivait une rencontre occidental-indigène où les enfants du progrès reconnaissaient enfin leur subordination et leurs erreurs. Parallèlement, ce discours général qui ne se fatiguait de redessiner la topographie des lieux et des peuples selon des valeurs morales symétriquement inverses à celles de la topographie du progrès et du développement, jouait un rôle essentiellement « contextuel ». En effet, sa fonction consistait à planter le décor de la rencontre, comme une sorte de discours d'accueil qui permet à tout le monde de se reconnaître comme faisant partie d'un même grand ensemble, d'une « communauté de discours » (Herzfeld 2005). Une fois les balises posées et la confiance installée, du moins en partie, un travail discursif et rituel plus proche du « fait-sur-mesure » pouvait prendre place.

Ainsi, un constat général et porteur pour l'ensemble des terrains a émergé: une version " universalisée », « standardisée », voire « canonisée » du chamanisme prend forme et se consolide dans ces lieux particuliers qui tiennent le rôle d'épicentres à l'intérieur des réseaux intercontinentaux. La ville d'Iquitos et celle de Cusco, au Pérou, ont été des terrains particulièrement éclairants à cet égard. Soutenus par des sites internet et des forums virtuels de discussion et de rencontre, des centres chamaniques et des chamanes particulièrement reconnus par un public itinérant et international étendent leur influence au sein des réseaux dans lesquels ils font figure d'autorité. Des discours et des pratiques déjà formatés au goût d'un public occidental, international et itinérant, s'actualisent en permanence au contact de ces clients et se rediffusent à travers eux vers les zones plus marginales de ces mêmes réseaux. L'autorité des milieux de culte en Europe et en Amérique du Nord occupe de ce point du vue un rôle subordonné dans cette logique de formatage des discours et des pratiques chamaniques.

Les pratiques rituelles liée à l'ingestion de plantes psychotropes semblaient être les plus transposables et constituent la composante rituelle d'origine amérindienne ayant le plus de succès international (Losonczy et Mesturini Cappo 2011). À côté des plantes psychoactives, les huttes de sudation ${ }^{3}$, la quête de vision associée au jeûne et l'utilisation de chants et d'instruments de musique divers, dont tout particulièrement le tambour d'origine sibérienne, forment un répertoire largement répandu et stabilisé

3. Également connues sous le terme lakota « inipi ». 
parmi le public chamanique occidentalisé et itinérant. Les pratiques diffusées par la Foundation for Shamanic Studies, fondée par Michael Harner, et celles qui caractérisent les rituels des Native American Churches jouent un rôle fondamental dans ce processus de sélection, de standardisation et de circulation des pratiques. Ceci dit, les chamanes qui ont fait l'objet de mon terrain ont la particularité de se distinguer de ces grandes " institutions » contemporaines du chamanisme et de prôner ainsi une interprétation plus individualisée et indépendante de leur pratique. Un nombre important des clients de ces mêmes chamanes avait néanmoins participé à des séances rituelles de l'un de ces deux grands ensembles chamaniques contemporains et ils les considéraient une étape parmi d'autres de leur parcours initiatique. Il en va de même pour les «Eglises de l'ayahuasca ${ }^{4} \gg$ qui se sont formées au Brésil et qui connaissent aujourd'hui un succès croissant: Santo Daime, União do vegetal et Barquinha. En adoptant une perspective itinérante, j'ai pu observer que même ces formes de chamanisme plus institutionnalisées et hiérarchisées s'inscrivaient dans des circuits chamaniques ouverts et mouvants, où la participation faisait le plus souvent objet d'un engagement temporaire voire éphémère. Les éléments les plus stables étaient, bien évidemment, les chamanes-fondateurs et les sommets des hiérarchies de chacune de ces institutions. Mais au-delà de ces noyaux durs, la démarche de la clientèle chamanique semblait guidée par une logique cumulative et par une mise à l'épreuve constante de l'efficacité des procédés et des experts rituels.

Le fait de réaliser un terrain en me mettant dans la même position qu'un pèlerin de l'initiation chamanique m'a aussi permis de suivre certains experts rituels en observant comment leur statut et leur compétence se transformaient de contexte en contexte. J'ai pu observer comment un chamane équatorien résidant en Italie du Nord et hautement réputé auprès d'un public essentiellement franco-italien a été traité de charlatan par des experts rituels péruviens lorsqu'il a guidé un groupe d'Européens lors d'un voyage initiatique en Equateur et au Pérou. Ce même chamane a été traité avec méfiance dans le cadre d'un festival chamanique d'envergure qui a eu lieu dans le sud de la France: son approche essentiellement « discursive » et peu « rituelle» du chamanisme remettait en cause ce que les autres chamanes présents définissaient comme chamanique. Accusé d'être « un hippie qui joue au gourou » plutôt que d'être « un vrai praticien-chamane », il a été exclu des festivals pour les années suivantes.

En passant trois fois trois mois dans la ville amazonienne d'Iquitos entre 2004 et 2006, j'ai eu l'occasion d'apercevoir comment l'afflux croissant de touristes et de pèlerins du chamanisme provenant tant des pays du Nord que des mégapoles sud-américaines créait des dynamiques complexes de concurrence, de discrédit et d'accusations croisées de sorcellerie parmi les experts locaux qui cherchaient à avoir accès à ce genre de réseau. La présence d'initiés occidentaux parmi les experts rituels locaux - élément souvent indispensable pour assurer un travail de traduction langagière et culturelle entre les praticiens amazoniens et la clientèle étrangère - contribuait à faire du chamanisme local une sorte d'interface ayant de multiples visages. Mais si l'alliance avec un gringo initié était la clé d'une entreprise chamanique florissante ainsi que d'un prestige international, elle allait le plus souvent de pair avec des accusations de « corruption » et de «perte de pouvoir » dans les réseaux locaux; accusations qui

4. Substance psychoactive tirée d'un végétal amazonien. 
pouvaient aboutir à l'exclusion du chamane de ses cercles sociaux habituels. Garder une réputation de chamane puissant dans les deux types de réseaux à la fois était une tâche difficile pour les experts amazoniens. La redistribution systématique des gains obtenus du travail avec les étrangers à l'intérieur du réseau des parents et clients locaux était une condition sine qua non pour maintenir avec succès des alliances chamaniques tant locales qu'internationales.

Mais parmi les « espaces » qui accueillent et supportent l'internationalisation du chamanisme, un « territoire » nouveau joue un rôle tout particulier: l'espace du réseau internet. Les sites qui surveillent l'utilisation des références, appellations et revendications chamaniques se multiplient ${ }^{5}$ et les organisations indigènes se soucient du copyright en dénonçant les fraudes et les emprunts illégitimes. Le terme plastic shaman est désormais monnaie courante sur la scène internationale du chamanisme et reproduit la sempiternelle distinction entre le médecin et le charlatan (Stengers 1995). L'argument le plus utilisé pour discréditer un «chamane » porte sur le caractère auto-attribué du titre et sur la revendication illégitime d'une appartenance ethnique. L'accusation de commercialiser des pratiques ethniques sans l'assentiment des ethnies concernées est omniprésente et soutenue par des individus qui se revendiquent, quant à eux, aptes à écrire sur le Net au nom des communautés concernées. La prétention à l'authenticité des chamanes affichés sur le Web et la légitimité chamanique de leurs détracteurs s'affrontent sans cesse dans une logique de méfiance et de discrédit permanents. À côté de ceux qui défendent un « chamanisme universel » et qui promeuvent les « savoirs traditionnels », on retrouve ceux qui proposent de défendre ces mêmes savoirs en dénonçant l'appropriation culturelle et, dans le cas des plantes psychotropes, la biopiraterie. L'affirmation "Native people do not use the label 'Shaman'» est souvent mise en avant afin d'éviter aux sympathisants des pratiques indigènes d'être dupes des « chamanes en plastique » mais elle manifeste aussi l'étendue de l'usage international et commercial de cette étiquette et la méfiance qui l'accompagne. Ces enjeux conflictuels restituent au chamanisme " globalisé » une dimension sociale importante qui rappelle des logiques plus locales d'encadrement et de contrôle de la figure du chamane et de ses pouvoirs..

L'affichage virtuel du chamanisme permet de percevoir la tension existant entre un chamanisme en voie de standardisation et des dynamiques de régulation sociale qui évoquent un fonctionnement acéphale, sans institution unique capable de normaliser le culte à partir d'un pouvoir central (Hamayon 2000, 2003). Si d'une part les discours se standardisent, d'autre part, la circulation d'anecdotes et de rumeurs dans les forums virtuels et la façon dont ceux-ci contribuent à construire ou à détruire la réputation d'un chamane, replonge ce phénomène chamanique a priori globalisé dans un univers fait d'alliances réelles et de conflits magiques et politiques, ainsi que d'accusations croisées de charlatanisme et de sorcellerie. Menacé en permanence par le discrédit, le " chamane globalisé ", tout comme le chamane pratiquant pour un public plus localement situé, ne maintient sa réputation et son pouvoir qu'en faisant preuve de performance discursive et rituelle dans le face-à-face avec une clientèle toujours prête à le discréditer. L'affichage internet et les interconnections qu'il implique semblent, en quelque sorte, le lieu privilégié d'un chamanisme en voie de canonisation. Mais

5. Voir par exemple: www.newagefraud.org ou www.religiousforums.com 
l'ethnographie des interactions concrètes et ponctuelles entre chamanes et clients internationaux débouche sur des hypothèses divergentes et complémentaires à celle d'une « universalisation du chamanisme ». Pour standardisés que les discours ou les séquences rituelles puissent être, lorsqu'un client demande une réponse à un problème personnel - qu'il soit pragmatique, psycho-émotionnel, " magique » ou un mélange des trois - des négociations complexes entre le chamane et le client s'enclenchent afin de co-construire un sens, un changement, une transformation, une efficacité ressentie comme telle par le client. Le besoin de performance et d'efficacité « sur mesure », et les dynamiques interactionnelles qui se mettent en place pour donner une forme à cette efficacité, exposent ces mêmes discours et pratiques à la subjectivité et à l'éphémère. Le caractère standardisé des discours et des pratiques apparaît ainsi comme une sorte de consensus de base, un scénario de compromis, un espace intellectuel apte à être partagé, à l'intérieur duquel des thématiques et des gestes plus spécifiques peuvent émerger. L'étude des échecs, des interactions problématiques et des «traductions impossibles » entre les univers de certains chamanes et de leurs clients a permis de développer et d'approfondir la tension existant entre discours et pratiques standardisés et interactions ponctuelles en touchant de près à des questions de " différence culturelle » et de " malentendu ritualisé » comme stratégie de communication dans des contextes interculturels (Losonczy et Mesturini Cappo 2013).

De plus, dans l'univers du discours chamanique universalisé, celui qui voudrait définir le chamanisme une fois pour toutes semble toujours se fonder sur un régime de légitimation qui met en tête de liste l'expérience vécue: intime, individuelle et incontestable. La " littérature chamanique » émergeante est un témoignage clair du statut donné à l'expérience individuelle et à son interprétation dans la légitimation de l'efficacité du chamanisme, de ses experts et de ses procédés. Or, l'existence même de ces récits expérientiels, leur circulation, leur aptitude à formater les attentes de nouveaux expérimentateurs démontrent la possibilité d'une expérience chamanique transposable et renouvelable. Ces récits semblent de ce fait remplir un rôle de «médiation », en ce sens qu'ils placent le représentant-médiateur en position de dépositaire du passé et de garant du futur, selon une logique que Bruno Latour définit comme « processuelle »:

La logique de procession ne progresse pas, sinon en intensité; elle craint
l'innovation bien qu'elle ne cesse continuellement d'inventer; elle s'efforce de
ne pas rabâcher bien qu'elle ne cesse continuellement de répéter les mêmes rites
(...) Elle aime par-dessus tout établir des correspondances, saturer de liaisons
transversales les différents messages amassés au cours des temps. Elle aime
épurer continuellement le message, mais chaque épuration devient un trésor
nouveau qui vient s'ajouter au dépôt sacré et l'enrichir, le compliquer encore (...)
Immense, vénérable, compliquée, infaillible, trahissante et traduisante, saturée
de médiations, telle est cette communauté qui maintient la tradition intacte en
l'enrichissant, en l'inventant de toutes pièces. (Latour 1990:46-49)

Les récits expérientiels demeurent performants tant que leur contenu reste toujours variable et actualisé. Si l'écrit semble figer l'expérience, l'essor de ce type de littérature, la multiplication faramineuse des ouvrages et sites internet ainsi que l'échange constant de récits expérientiels lors des interactions, ritualisées ou pas, qui peuplent les routes du chamanisme, restituent au phénomène un caractère changeant et multiforme.

Le discours « chamanique » qui circule et qui fait circuler s'inscrit ainsi dans un paysage composé conjointement par les itinéraires et circuits initiatiques et par 
les innombrables récits expérientiels qui retracent les voyages oniriques vécus par les praticiens, les initiés ou les expérimentateurs occasionnels. Décrire ce paysage chamanique contemporain en analysant les récits et discours qui circulent sous l'étiquette chamanique nous confronte à un mouvement constant, où des récits emblématiques formatent des expériences ponctuelles et où des expériences ponctuelles engendrent de nouveaux récits emblématiques.

À l'intérieur de cet espace du discours chamanique performatif, la notion de « limite » joue un rôle particulier. Qu'il s'agisse de voyages physiques ou oniriques, le but de cet ethos de l'itinérance est de toucher, voire de franchir, les limites de l'imaginaire, les limites du psychisme et les limites culturelles. L'itinérance prônée par les réseaux chamaniques semble justement vouloir faire converger ces trois façons " d'aller au-delà » dans un seul et même voyage. Dépaysement culturel, thérapie psychique et expérimentation mystico-spirituelle sont appelés à un même rendez-vous ritualisé et expérientiel. C'est en ce sens qu'apparaît la pertinence d'une approche « topographique » du chamanisme contemporain: une approche qui retrace, dessine et compare les différents espaces et géographies créés par l'itinérance à étiquette chamanique et qui lui servent de support.

Les liens entre chamanisme et itinérance suggèrent différentes perspectives de recherche et d'analyse. Les ethnologues du chamanisme qui, dans différents contextes, ont mis en avant l'importance de la dimension itinérante dans les chamanismes d'hier et d'aujourd'hui, éclairent la différence de prisme de lecture qui existe entre les anthropologues du chamanisme universalisé et ceux qui s'en différencient.

\section{Itinérance et topographies}

Un ensemble de discours, de pratiques et d'écrits semble constituer, entre la fin des années 1960 et le début des années 1990, le dénominateur commun d'un « chamanisme » désormais sorti tant des cercles universitaires que des peuples supposés l'incarner. Ce dénominateur commun se compose d'un intérêt pour des systèmes religieux lointains, de la volonté d'affirmer la supériorité de traditions spirituelles « d'ailleurs »par rapport aux institutions religieuses d'origine chrétienne, de l'omniprésence d'un désir de dissidence allant contre toute autorité préexistante et de l'ouverture d'un champ d'expérimentation tant personnelle que clinique portant sur de nouvelles techniques thérapeutiques et sur l'utilisation de substances psychotropes.

La démonstration de l'universalité du chamanisme, de son efficacité thérapeutique et de son utilité pratique pour «l'évolution de la conscience » de l' « homme occidental» et de la "société globale », devient la pierre de touche de cette nouvelle approche. L'engagement pragmatique de certains intellectuels, dont de nombreux anthropologues, porteurs du mouvement - notamment Carlos Castaneda, Michael Harner, Jeremy Narby, Stanislav Grof, Ralph Metzner, Terence Mc Kenna, Luis Eduardo Luna - dans la défense des « peuples premiers », dans des projets écologiques divers, dans la création d'ONGs et de centres de formation aux nouvelles spiritualités, ou encore dans l'ouverture de centres thérapeutiques expérimentaux utilisant des substances psychotropes, fait sortir du monde académique bon nombre de ces personnages devenus emblèmes d'un changement des mentalités. Leurs sympathisants et apprentis constituent à la fois leur public et la source de financement de leurs recherches et projets. Parallèlement, certaines figures d'origine indigène, principalement nord-américaines, s'inscrivent 
avec succès dans la littérature chamanique émergeante en se présentant comme les représentants légitimes de leur groupe. Les « chamanes » deviennent ainsi « auteurs ». Leurs ouvrages, qui portent sur leur « cosmologie », leurs « rituels » et leur vision du monde, s'adressent aux « frères pâles » en même temps qu'ils deviennent un moyen de légitimation et de reconnaissance pour le monde indigène. .

Progressivement, le « chamanisme » tel qu'utilisé dans les réseaux internationaux devient un champ théorico-pratique censé témoigner de l'existence d'une tradition spirituelle unique, primordiale et universelle - ce qui n'est pas sans rappeler l'entreprise théorique de Joseph-François Lafitau: « démontrer l'universalité et le caractère originel du monothéisme en dépit de dégénérescences » (Menget 2002:330). L'ouvrage de référence à cet égard est sans aucun doute Le chamanisme et les techniques archaïques de l'extase de Mircea Eliade, dont l'omniprésence pourrait presque en faire la «bible» d'un chamanisme en voie d'universalisation. Bien que les porteurs du mouvement se révolteraient face à la comparaison du chamanisme universalisé avec le monothéisme, le parallèle tient bien la route si l'on se penche sur cette attitude, propre au chamanisme universalisé, qui prône la convergence des traditions, croyances, entités spirituelles et pratiques rituelles les plus diverses vers une seule et même origine et finalité. Ce noyau dur peut avoir des appellations diverses mais renvoie systématiquement à l'idée d'un principe vitaliste ou d'une « conscience universelle » propre à tout être vivant. Les incroyants, les sceptiques, les désenchantés, les défenseurs athées de la modernité et du progrès qui ne reconnaissent pas le besoin de se " reconnecter " avec cette sagesse primordiale font exception à cette universalité mais y sont intégrés grâce à une taxinomie évolutionniste inversée qui fait d'eux les " primitifs » du développement spirituel de l'humanité.

Aux habitants des régions reconnues comme « authentiquement chamaniques », on demande d'incarner ce chamanisme universalisé et on fait de leur caractère chamanique le socle de la reconnaissance de leur « culture ». Le glissement toujours possible entre religion, culture et ethnie qui caractérise la discipline anthropologique se retrouve dans l'édifice théorique du chamanisme universalisé, ce qui témoigne des liens étroits qui existent entre leurs développements respectifs. Les discours et les convictions du chamanisme universalisé portent clairement la marque de la contre-culture et redessinent, en sens contraire, la géographie dictée par l'idéologie du progrès. Selon Renée de la Torre Castellanos:

dans le cas de la spiritualité New Age, les connexions sont allées en sens inverse des pôles d'attraction du progrès néolibéral. Le New Age correspondant à une sensibilité postmoderne et contre-culturelle, les aventuriers de la quête spirituelle ont recherché des expériences mystiques dans des horizons culturels reniés ou distancés par la modernité: les horizons oriental, indigène, naturel. (2011:145)

Afin de se démarquer de ce prisme performatif et universalisant de la notion de chamanisme, d'autres anthropologues recentrent leur perspective sur le modus operandi du chamanisme tout en posant, conjointement, la question de la pertinence d'une utilisation transcontextuelle et comparative de cette notion. Ils posent la question du chamanisme en termes d'interconnexion des peuples et d'échange des pratiques.

Ainsi, pour Manuela Carneiro da Cunha, le pouvoir chamanique est la conséquence de voyages aux limites de l'ordinaire, qu'il s'agisse de voyages terrestres ou oniriques. Cet auteur soutient que dès le début de la période coloniale, les chamanes amazoniens 
entretenaient, dans la plupart des cas, des clientèles à une échelle régionale, toutes origines ethniques confondues (Carneiro da Cunha 1998: 7-22). Anne-Marie Losonczy décrit comment, parmi des Embera, l'apprenti-chamane doit, par coutume, accomplir une série "d'itinérances didactiques " auprès des chamanes d'ethnies voisines et parfois auprès de chamanes urbains. Pour légitimer son pouvoir auprès des siens, le jeune chamane doit entreprendre un voyage afin aller étudier les procédés rituels des Autres. L'itinérance des apprentis chamanes est une caractéristique indispensable à l'apprentissage de la compétence chamanique. La rencontre avec l'extérieur devient un moyen privilégié d'accumulation de savoirs et de pouvoirs susceptibles de bénéficier à la communauté d'origine (Losonczy 2006).

Le point de vue que le chamane acquiert grâce à ses voyages est, d'après Manuela Carneiro du Cunha, une perspective destinée à rester parcellaire, biaisée, incomplète et partiale. Le but du chamane itinérant ne serait point de construire une vision abstraite, ordonnée et universalisée du monde mais bien d'aller aux limites de son monde, et donc de celui de la communauté pour laquelle il opère, afin de le compléter et de le complexifier avec des éléments puisés au-delà des frontières, éléments qui se révèlent opérants tant pour le maintien de l'ordre interne que pour les relations avec ce qui est à l'extérieur. Son monde est celui auquel il appartient et où il a tissé ses liens les plus proches, un monde ayant un emplacement spécifique et déterminant, un monde auquel il va revenir, au service duquel il va travailler et pour lequel il a accompli ce voyage.

Ao longo de suas viagens a outros mundos, ele observa sob todos os ângulos, examina minuciosamente e abstém-se cuidadosamente de nomear o que vê. Donde a suspensão da linguagem ordinária, substituída por essas "palavras torcidas" [...]. como se escrutasse por apalpadelas, como se abordasse um domínio desconhecido cujos objetos só se deixam ver parcialmente, o xamã adota uma limguagem que expressa um ponto de vista parcial. (Carneiro da Cunha 1998:13) ${ }^{6}$

Sa tâche serait ainsi d' "interpretar o inusitado, conferir ao inédito um lugar inteligível, uma insersão na ordem das coisas $»{ }^{7}$. Dans cette incorporation progressive d'éléments nouveaux, le chamane devient un traducteur qui ne se limite pas à mettre de nouveaux mots sur des objets anciens. Il traduit afin de réaménager ses espaces et ses repères, afin d'apprivoiser la nouveauté et de la rendre pensable et intelligible. Le " chamane-traducteur » devient un personnage " idealmente situado para encarnar a contento o projeto de juncão do local e do global $»^{8}$. Dans cette idée de «projet » chamanique articulant le local et le global, la notion de « global » n'a de sens que lorsqu'elle sert à désigner cette grande inconnue " extérieure », floue et mobile, qui tout comme le monde des invisibles des Embera (Losonczy 2006), doit être apprivoisée

6. Traduction: «Au cours de ses voyages dans d'autres mondes, il observe sous tous les angles, il examine minutieusement et il s'abstient soigneusement de nommer ce qu'il voit. Là où le langage ordinaire est suspendu, remplacé par des " paroles tordues » [...] comme s'il scrutait en tâtonnant, comme s'il abordait un domaine inconnu dont les objets ne se laissent voir que partiellement, le chamane adopte un langage qui exprime un point de vue partiel ».

7. Traduction: « interpréter l'inhabituel, conférer à l'inédit un lieu intelligible, une insertion dans l'ordre des choses ».

8. Traduction : « situé de manière idéale pour incarner à volonté le projet de jonction du local et du global ». 
afin que certaines de ses composantes, capturées, fixées et nommées, puissent entrer et faire partie d'un paysage culturel soutenu par une «topographie du connu » faite sur mesure pour un contexte spécifique. En effet, le travail de ce chamane voyageurtraducteur, garant et négociateur des limites du monde, ne s'accomplit que moyennant la confrontation, voire la contestation, qui naît de l'interaction avec ceux pour qui il traduit.

Comment poser la question de la continuité entre le chamanisme " ethnique », le chamanisme « métis » et le chamanisme « touristique » proposé pour et par des « occidentaux»?

Ethnographier la continuité de l'itinérance nous permet de penser autrement les ruptures et les différences présentes dans le contexte amazonien. Cela permet aussi de remettre en cause une idée préconçue très répandue qui veut que toute forme de chamanisme soit le produit d'une adaptation progressive et continue de savoirs et de pratiques indigènes qui, émergeant constamment de la forêt, s'adapterait au fur et à mesure aux nouveaux contextes urbains.

En considérant l'itinérance chamanique comme véhiculant l'entrée d'éléments extérieurs nouveaux, il devient légitime de penser qu'une période caractérisée par une série de changements majeurs, telle la période coloniale, aurait accru la longueur et l'échelle des itinérances. D'après Manuela Carneiro, nombreux seraient les exemples sur tout le continent latino-américain qui permettent d'affirmer l'existence d'une croissance particulière du chamanisme, associée à l'écroulement des institutions politiques et économiques de type traditionnel. En Amazonie en particulier, ce chamanisme fleurissant, désormais lié au système d'endettement et à l'exploitation massive du caoutchouc, s'organiserait selon des modalités nouvelles et des itinéraires différents de ceux du passé. Pour ce qui est du chamanisme ayahuasquero ${ }^{9}$ en particulier, très répandu dans l'ensemble de l'Amazonie occidentale, Peter Gow remet en cause l'idée selon laquelle ce type de pratiques appartiendrait à une tradition précolombienne, restée plus ou moins intacte selon les cas:

I argue that ayahuasca shamanism has been evolving in urban contexts over the past three hundred years, and that it has been exported from these towns to isolated tribal people to become the dominant form of shamanic curing practice in the region. It evolves as a response to the specific colonial history of western Amazonia and is absent precisely from those few indigenous people who were buffered from the process of colonial transformation caused by the spread of the rubber industry in the region. (Gow 1994:91)

Ainsi, l'étude des phénomènes concrets de circulation, d'emprunt et de dialogue qui prennent forme autour des pratiques rituelles vernaculaires nous permet d'apercevoir des itinéraires, des rapports de pouvoir et des dynamiques relationnelles complexes susceptibles de déconstruire les oppositions binaires qui départagent modernité et tradition, acculturation et résistance, cultures ethniques et culture occidentale. Ces dynamiques relationnelles révèlent d'enjeux d'influence complexes où la technologie et la magie jouent des rôles de premier ordre dans des logiques de fascination et d'influence réciproques.

9. Type de ritualité chamanique qui suppose l'ingestion ritualisée d'une substance psychotrope d'origine amazonienne, connue internationalement sous le terme quetchua «ayahuasca». 
La continuité de l'itinérance permet de tracer un lien au-delà des ruptures historiques et contextuelles. Cette itinérance apparaît comme un modus vivendi du chamanisme et de ses représentants qui suscite et prône une sélection continue et négociée des pratiques et discours retenus comme performants dans le cadre d'interactions rituelles ponctuelles constamment renouvelés. Le contact entre les peuples dans des contextes et à des moments historiques donnés produit des sélections plus ou moins collectives et standardisées ou, au contraire, ponctuelles et éphémères. Si le "chamanisme métis » peut apparaître comme une « transformation commune » qui s'est stabilisée au contact avec le catholicisme populaire et la violence de la période coloniale et postcoloniale (voir Langdon dans ce même volume), le chamanisme international et transcontinental pose le défi d'une nouvelle sélection en voie de stabilisation. Pour déterritorialisée et décontextualisée qu'elle puisse paraître, cette nouvelle forme reproduit des dynamiques de contrôle social de la réputation et du pouvoir chamanique ainsi qu'une mise à l'épreuve ritualisée de l'efficacité pragmatique qui déterminent « par le bas » les objets et les modalités d'une circulation d'échelle globale.

\section{Quelques conclusions}

L'étude des discours de présentation et de défense d'un chamanisme unique et primordial ainsi que la diffusion de séquences rituelles de plus en plus uniformes, leurvaste représentation sur le réseau virtuel et leur omniprésence dans les divers terrains réalisés nous confrontent avec un chamanisme en voie de standardisation et d'universalisation. Mais au-delà de ces aspirations universalistes, si l'on pose la question de l'utilité de ce type de discours - et plus spécifiquement, de l'usage et de la place qui lui sont réservés par les centres chamaniques amazoniens, les chamanes sud-américains itinérants ou les chamanes européens initiés aux pratiques amérindiennes -, on s'aperçoit qu'il sert à créer un terrain globalisé d'entente. En balisant ce terrain de façon à ce qu'il puisse être le plus vaste possible, le chamanisme universalisé ouvre un champ relationnel et un espace d'entente où l' « Occidental » et l'« Indigène » jouent le rôle de frontières ultimes et emblématiques (Losonczy et Mesturini 2010). Ce chamanisme redéfinit une géographie globale tout en ouvrant un champ d'expérimentation initiatique et onirique où la possibilité d'un voyage individuel est possible. Entre l'universel et l'individuel, l'impact des structures sociales et des sociabilités émerge à travers le processus constant de négociation des anciennes et nouvelles alliances dans des lieux où les réseaux locaux et les voyageurs transcontinentaux entrent en contact.

L'ethnographie itinérante s'est révélée pertinente pour observer comment de nouvelles formes chamaniques, plus standardisées et mobiles, s'ancrent (ou se ré-ancrent) dans des lieux spécifiques; elle permet aussi de saisir les dynamiques interactionnelles qui prennent place dans ces différents cadres. L'approche itinérante permet aussi, comme nous l'avons suggéré, de poser l'hypothèse d'un chamanisme universalisé qu'il serait possible d'ethnographier en tant que phénomène à part entière tout en interrogeant les continuités et les ruptures qui le relient à des formes plus vernaculaires ou plus localisées. Parallèlement, la question de l'itinérance émerge comme une composante des chamanismes sud-américains d'hier et d'aujourd'hui et permet de penser une continuité entre des fonctionnements plus vernaculaires et le processus de globalisation contemporain. 
Les voyages du chamane, l'interprétation qu'il en fait et la perspective spatiale qui en découle s'inscrivent dans un langage topographique qui découpe les espaces selon des logiques relationnelles plurielles, où tant les entités invisibles que les voisins proches et lointains trouvent leur sens et leur place. La rencontre emblématique indigène-occidental peut ainsi être comprise comme une expression de ce même langage topographique qui nomme et pose dans l'espace environnant des repères relationnels toujours renégociables. Le caractère relationnel de cette approche topographique, qui génère un constant appel de références à l'altérité, permet d'expliquer son aptitude à circuler et à faire circuler, malgré les changements d'échelle et les différences culturelles. Ainsi, la démarche topographique et la facette itinérante des pratiques chamaniques vernaculaires leur aurait permis, depuis toujours, d'entrer en circulation et de participer avec succès à une mise en réseau, d'abord locale et coloniale, puis postcoloniale et globalisée.

\section{Références citées}

CARneiro da Cunha, Manuela, 1998. «Pontos de vista sobre a floresta amazónica: xamanismo e traduçao », Mana, 4 (1): 7-22.

Chaumeil, Jean-Pierre, 2003 [1992]. " Chamanismes à géométrie variable en Amazonie », in Roberte Hamayon (dir.), Chamanismes, 159-176. Paris: Presses Universitaires de France.

DE la Torre Castellanos, Renée, 2011. «Les rendez-vous manqués de l'anthropologie et du chamanisme », Archives de sciences sociales des religions, $153: 145-158$.

Devereux, Georges, 1970. Essais d'ethnopsychiatrie générale. Paris : Gallimard.

Gow, Peter, 1991. Of Mixed Blood. Kinship and History in Peruvian Amazon. Oxford : Clarendon Press.

-, 1993. «Gringos and Wild Indians. Images of History in Western Amazonian Cultures », L'Homme, 33, (126-128) : 327-347.

-, 1994. « River People: Shamanism and History in Western Amazonia », in Nicholas Thomas et Caroline Humphrey (éds), Shamanism, History and the State, 90-114. Ann Arbor: University of Michigan Press.

Hamayon, Roberte, 2000. «Avant-Propos » in Denise Aigle, Bénédicte Brac de la Perrière, Jean-Pierre Chaumeil (éds), La politique des esprits. Chamanismes et religions universalistes, 7-13. Nanterre: Société d'Ethnologie.

-, 2003. «Introduction à Chamanismes. Réalités autochtones, réinventions occidentales », in Roberte Hamayon (dir.), Chamanismes, 7-54. Paris : Presses Universitaires de France.

Hammer, Olav, 2001. Claiming Knowledge. Strategies of Epistemology from Theosophy to the New Age. Leiden: Brill.

HanegraAf, Wouter J., 1996. New Age Religion and Western Culture. Esotericism in the Mirror of Secular Thought. Leiden: Brill.

Herzfeld, Michael, 2005. Cultural Intimacy. Social Poetics and the Nation-State. New York et Londres: Routledge.

LANGDON, Esther Jean, 2007. «Shamans and Shamanisms: Reflections on Anthropological Dilemmas of Modernity », Vibrant, 4 (2):27-48.

LATOuR, Bruno, 1990. « Quand les anges deviennent de bien mauvais messagers », Terrain, 14:76-91.

LÉVI-Strauss, Claude, 1973. Anthropologie structurale II. Paris: Plon.

Losonczy, Anne-Marie, 1990. «La maîtrise du multiple. Corps et espace dans le chamanisme embera du Choco (Colombie)», L'Homme, 114, avril-juin, 30 (2): 75-100. 
-, 2006. Viaje y violencia. La paradoja chamánica embera. Bogota: Universidad Externado de Colombia.

Losonczy, Anne-Marie et Silvia Mesturini Cappo, 2010. « Entre 1' 'Occidental' et 1' Indien’. Ethnographie des routes du chamanisme ayahuasquero entre Europe et Amériques », Autrepart, 56:93-110.

-, 2011. « Pourquoi l'ayahuasca ? De l'internationalisation d'une pratique rituelle amérindienne », Archives de sciences sociales des religions, $153: 207-228$.

-,2013 (sous presse). « Ritualized Misunderstanding between Uncertainty, Agreement and Rupture: Communication Patterns in Euro-American Ayahuasca Ritual Interactions », in Beatriz Caiuby Labate et Clancy A. Cavnar (éds), The Expansion and Reinvention of Ayahuasca Shamanism. Oxford: Oxford University Press.

Menget, Patrick, 2002. « Histoire de l'Anthropologie » in Pierre Bonté et Michel Izard (éds), Dictionnaire de l'ethnologie et de l'anthropologie, 328-332. Paris: Presses Universitaires de France.

Mesturini, Silvia, 2010. Espaces chamaniques en mouvement: itinéraires vécus et géographies multiples entre Europe et Amérique latine. Thèse doctorale présentée à l’Université Libre de Bruxelles.

Stengers, Isabelle, 1995. «Le médecin et le charlatan » in Tobie Nathan et Isabelle Stengers (éds), Médecins et sorciers, 115-161. Paris: Les empêcheurs de penser en rond.

TAussig, Michael, 1986. Shamanism, Colonialism and the Wild Man. A study in Terror and Healing. Chicago: University of Chicago Press.

Thomas, Nicholas et Caroline Humprey, 1994. Shamanism, History and the State. Ann Arbor : University of Michigan Press. 